\title{
Optoelectronic Oscillator for Arbitrary Microwave Waveform Generation
}

\author{
Yang Chen, Member, IEEE, Member, OSA, Pengcheng Zuo, Taixia Shi, Graduate Student Member \\ IEEE,
}

\begin{abstract}
An optoelectronic oscillator (OEO) for arbitrary microwave waveform generation based on Fourier domain mode-locking (FDML) is proposed. A reconfigurable optical waveform generator (OWG) is implemented via external electro-optical modulation, which serves as the optical source for the OEO. The optical waveform from the OWG can be seen as a series of frequency-scanning optical wavelengths in the frequency domain, and at any instant, the number of wavelengths can be from 0 to infinite. By setting the period of the optical waveform or its multiple equal to the cavity round-trip time, an FDML OEO implemented by a frequency-scanning microwave photonic filter is realized based on the phase-modulation-to-intensity-modulation conversion using a phase modulator cascaded with a phase-shifted fiber Bragg grating with its scanning characteristics according to the optical waveform, which can be utilized to generate low phase noise arbitrary microwave waveform using the inherent low phase noise merit of an OEO. A proof-of-concept experiment is performed. Linearly-chirped microwave waveforms and non-linearly-chirped microwave waveforms with a scanning bandwidth of $600 \mathrm{MHz}$, 2-level frequency hopping (FH) microwave waveform with an FH range of $400 \mathrm{MHz}$, and 218-bit binary and quaternary phase-coded microwave waveforms are generated from the OEO. To the best of our knowledge, this is the first time that an OEO is proposed for arbitrary microwave waveform generation.
\end{abstract}

Index Terms-Optoelectronic oscillator, arbitrary microwave waveform generation, Fourier domain mode locking, microwave photonic filter.

\section{INTRODUCTION}

$\mathrm{C}$ ONVENTIONALLY, microwave and millimeter-wave waveforms are generated in the electrical domain directly, but the frequency and bandwidth are usually limited due to the electronic bottleneck [1]-[3]. Photonic generation of microwave and millimeter-wave waveforms can overcome these problems, taking the

Manuscript received Feb. 4, 2021. This work was supported in part by the National Natural Science Foundation of China under Grant 61971193, in part by the Natural Science Foundation of Shanghai under Grant 20ZR1416100, in part by the Open Fund of State Key Laboratory of Advanced Optical Communication Systems and Networks, Peking University, China, under Grant 2020GZKF005, in part by the Science and Technology Commission of Shanghai Municipality under Grant 18DZ2270800, and in part by the Fundamental Research Funds for the Central Universities. (Corresponding author: Yang Chen.)

The authors are with the Shanghai Key Laboratory of Multidimensional Information Processing, East China Normal University, Shanghai 200241, China, and also with the Engineering Center of SHMEC for Space Information and GNSS, East China Normal University, Shanghai 200241, China (e-mail: ychen@ce.ecnu.edu.cn). inherent advantages of large bandwidth and high frequency offered by modern photonics [4]-[6]. Many photonic-based approaches [7] have been proposed to generate microwave and millimeter-wave waveforms during the past two decades. Among them, the optoelectronic oscillator (OEO) [8] has attracted great attentions because it can generate high-quality microwave or millimeter-wave waveforms with low phase noise. However, limited by the long mode built-up time, the conventional OEO can only be used to generate the single-frequency signal.

In 2017, a novel frequency-scanning OEO [9] was proposed by embedding a frequency-scanning microwave filter with its scanning period equal to the cavity round-trip time, in which a linearly-chirped microwave waveform (LCMW) with a chirp rate of $156 \mathrm{MHz} / \mu \mathrm{s}$ and a scanning bandwidth of $1 \mathrm{GHz}$ was generated. However, an unavoidable fact is that it is complicated to directly manipulate the scanning characteristics of the filter in the electrical domain. Subsequently, a frequency-scanning OEO [10] based on Fourier domain mode-locking (FDML) technique [11] was proposed, in which a frequency-scanning microwave photonic filter (MPF) based on the phase-modulation-to-intensity-modulation (PM-to-IM) conversion using a phase modulator cascaded with a phase-shifted fiber Bragg grating (PS-FBG) was constructed in the OEO in conjunction with a wavelength scanning optical waveform. An LCMW with a chirp rate of $0.34 \mathrm{GHz} / \mu$ s and a time-bandwidth product (TBWP) of 166650 was generated. The key to implementing an FDML OEO is to construct a frequency-scanning filter used for the mode selection in the electrical domain, whose scanning period or its multiple is equal to the cavity round-trip time. In this way, each cavity mode is synchronizing with the frequency scanning filter so that these modes do not have to rebuild from noise, which breaks the limitation of the time required for mode establishment [10]. Based on this principle, FDML OEOs using various linear frequency-scanning filters have been proposed to generate LCMWs during the past three years. In [12], an FDML OEO based on optically injected semiconductor laser and period-one oscillation was proposed, in which an equivalent frequency-scanning MPF was constructed by introducing an "injection strength controller" to adjust the injected optical power into the slave laser. An LCMW with a chirp rate of 180 $\mathrm{MHz} / \mathrm{ns}$ and a scanning bandwidth of $7 \mathrm{GHz}$ was generated. However, the frequency stability and the frequency scanning linearity are not good due to the unstable period-one oscillation. FDML OEOs for single-chirp LCMW generation based on stimulated Brillouin scattering (SBS) effect [13], [14] were also proposed, in which PM-to-IM conversion using the SBS gain spectrum [13] or the SBS loss spectrum [14] was realized to achieve the MPF in the OEO cavity. However, the main problems in [13] and [14] are that not only the frequency stability of the generated LCMWs will deteriorate, but also the operation frequency range of the generated LCMWs is 
difficult to precisely set due to the use of two independent laser sources. To solve these problems, an SBS-based FDML OEO using a single laser source and the carrier-suppressed single-sideband (CS-SSB) modulation was proposed in [15], resulting in good frequency-scanning linearity and an output frequency range that can be precisely set. In addition to FDML OEOs for single-chirp LCMW generation, some FDML OEOs for dual-chirp LCMW generation have been developed by constructing a frequency-scanning dual-passband MPF in the OEO cavity. An FDML OEO using two lasers driven by opposite triangle-wave currents was proposed for dual-chirp LCMW generation [16]. However, an electrical bandpass filter is essential to filter out the undesired interfering beating component between the two optical carriers. A polarization manipulated FDML OEO also using two lasers driven by two electrical sawtooth currents with opposite slopes was proposed for dual-chirp LCMW generation [17]. The polarization manipulation of the optical signals can avoid the problems in [16]. However, the methods in [16] and [17] have the problem that the frequency stability and frequency scanning linearity may be deteriorated due to the use of two independent laser sources. In [18], an SBS-based FDML OEO using a single laser and carrier-suppressed double-sideband (CS-DSB) modulation was proposed to generate complementary LCMW pairs, in which good frequency-scanning linearity was achieved. To generate LCMWs with even higher frequencies, the frequency-multiplied LCMW generation based on an FDML OEO [19] had been proposed by using a wavelength-scanning optical waveform generated through high-order nonlinear electro-optical modulation. Compared with the input frequency-scanning signal, frequency-multiplied LCMWs were obtained. It should be pointed out that both the methods in [12] and [17] can also generate frequency-multiplied LCMWs, but the frequency multiplication is realized out of the OEO cavity. Note that most of the methods mentioned above only focus on how to realize different FDML OEOs based on different designs for generating single-chirp LCMWs [12]-[15], dual-chirp LCMWs [16]-[18], and frequency-multiplied LCMWs [12], [17], [19]. Few works focused on the improvements in terms of cost, size, and performance until an FDML OEO without using a tunable signal source was proposed in [20]. All in all, either a frequency-scanning electrical bandpass filter or a frequency-scanning MPF constructed by a wavelength scanning optical waveform is needed for the realization of an FDML OEO. For the methods using an MPF, the LCMW with low phase noise is generated by converting the wavelength scanning optical waveform to the electrical domain with the help of a frequency-scanning OEO based on FDML.

However, until now, all the FDML OEOs are only reported to generate LCMWs. In this paper, for the first time, we propose an arbitrary microwave waveform generation approach based on an FDML OEO. A reconfigurable optical waveform generator (OWG) is constructed via optical CS-SSB modulation. The optical waveform from the generator can be seen as a series of frequency-scanning optical wavelengths in the frequency domain, and at any instant, the number of wavelengths can be from 0 to infinite. When the optical waveform serves as the optical source for an OEO based on the PM-to-IM conversion using a phase modulator cascaded with a PS-FBG, a frequency-scanning MPF with scanning characteristics according to the optical waveform is realized. By setting the period of the optical waveform or its multiple equal to the cavity round-trip time, an FDML OEO that can generate arbitrary microwave waveform is realized. LCMWs and non-linearly chirped microwave waveforms (NLCMWs) with a scanning bandwidth of $600 \mathrm{MHz}$, 2-level Frequency hopping $(\mathrm{FH})$ microwave waveform with an $\mathrm{FH}$ range of $400 \mathrm{MHz}, 218$-bit binary and quaternary phase-coded microwave waveform are generated from the $\mathrm{OEO}$ with a fiber length of about $200 \mathrm{~m}$ and $4.1 \mathrm{~km}$, respectively. To the best of our knowledge, this is the first time that a frequency-scanning OEO is proposed for arbitrary microwave waveform generation. The proposed method provides a promising solution to utilize the low phase noise merit of an OEO to generate low phase noise arbitrary microwave waveforms.

\section{PRINCIPLE}

A. Comparison Between the Standard Mode-Locked OEO and the FDML OEO

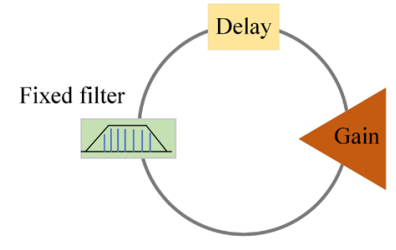

(a) Simplified model of ML-OEO

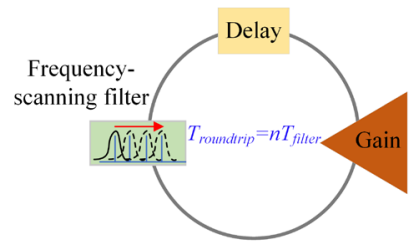

(b) Simplified model of FDML-OEO
Fig. 1. A simplified model to show the operation of a standard mode-locked OEO and an FDML OEO. (a) A standard mode-locked OEO, where multiple modes are simultaneously active and oscillating, (b) an FDML OEO, multiple modes are active and stored in the cavity, but the modes are oscillating one by one due to the frequency-scanning filter.

The FDML is a supplement to the standard mode-locking [11]. Applying the two different mode-locking techniques to an OEO, a standard mode-locked OEO [21] and an FDML OEO can be obtained, respectively. Fig. 1 displays the simplified models to show the operations of a standard mode-locked OEO and an FDML OEO. Simultaneous oscillation of multiple modes is realized in the standard mode-locked OEO, namely electrical frequency combs, as shown in Fig. 1(a). The condition of simultaneous oscillation of multiple modes will not change over time, which is a steady-state operation. However, the FDML technique is a quasi-steady-state operation, as shown in Fig. 1(b). The key to an FDML OEO is to generate a frequency-scanning filter for mode selection in the electrical domain, whose scanning period or its multiple is equal to the cavity round-trip time. In this way, each cavity mode is synchronizing with the frequency scanning filter so that these modes do not have to rebuild. That is to say, multiple modes are stored in the OEO cavity at the same time. During a scanning period of the frequency-scanning filter, these modes stored in the cavity appear one after another, as if these modes are oscillating at the same time. Since multiple modes are active at the same time and stored in the cavity, each of them does not need to be re-established from the noise, which breaks the limitation of the time required for mode establishment [10]. 


\section{B. Two Different Implementations for FDML OEO}

(a)

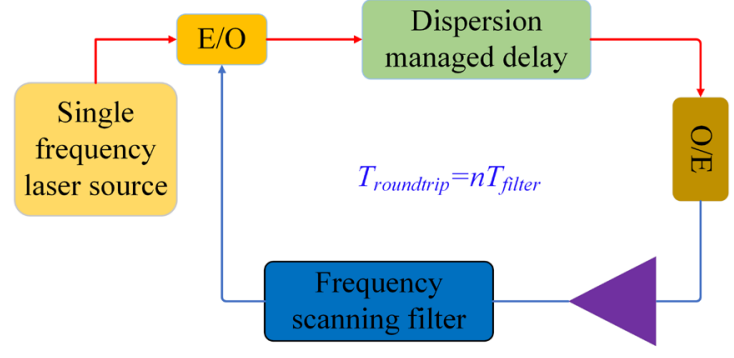

Gain medium

(b)

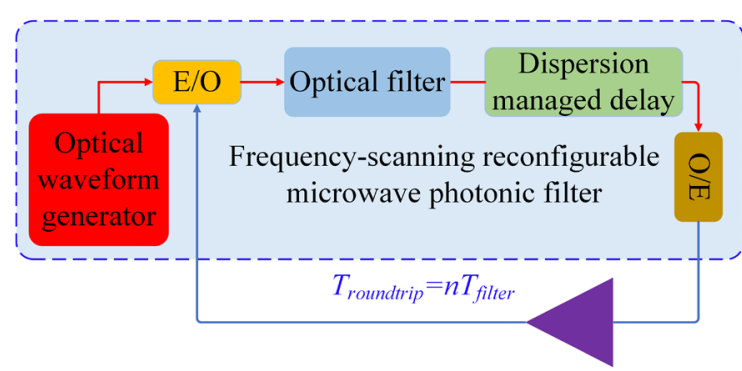

Gain medium (c)

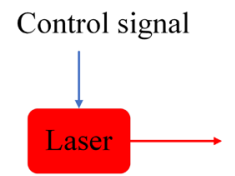

(d)

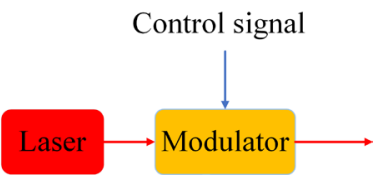

Fig. 2. Two implementation schemes of the FDML OEO. (a) The FDML OEO based on a single-frequency laser source and a frequency-scanning electrical filter, (b) the FDML OEO based on an optical waveform generator and a reconfigurable frequency-scanning MPF. Optical waveform generator based on (c) directly modulating a laser or (d) external modulation.

Considering the implementation of the FDML OEO, it can be roughly divided into two categories. As shown in Fig. 2(a), a single-frequency laser source and a frequency-scanning electrical filter with its scanning period or its multiple equal to the cavity round-trip time are employed to implement an FDML OEO. Fig. 2(b) shows the FDML OEO based on a frequency-scanning MPF with reconfigurable frequency-scanning characteristics using an $\mathrm{OWG}$ and an optical filter. A frequency-scanning MPF, based on the PM-to-IM conversion using a phase modulator cascaded with an optical filter, is constructed for mode selection. By setting the period of the optical waveform or its multiple equal to the cavity round-trip time, an FDML OEO with its scanning characteristics according to the optical waveform can be realized.

However, it is very complicated to directly manipulate the scanning characteristics of the filter in the electrical domain. Compared with the frequency-scanning electrical filter in Fig. 2(a), the frequency-scanning MPF in Fig. 2(b) is determined by the optical waveform from the OWG, which is much easier to reconstruct. In addition, for the second implementation in Fig. 2(b), there are usually two different methods to implement the OWG, i.e., directly modulating a laser or using external modulation, as shown in Fig. 2(c) and (d), respectively. From the perspective of providing an optical source for the FDML OEO, there is no essential difference between external modulation and direct modulation without considering the modulation bandwidth.

\section{Time-Varying Multi-Passband Frequency-Scanning Filter}
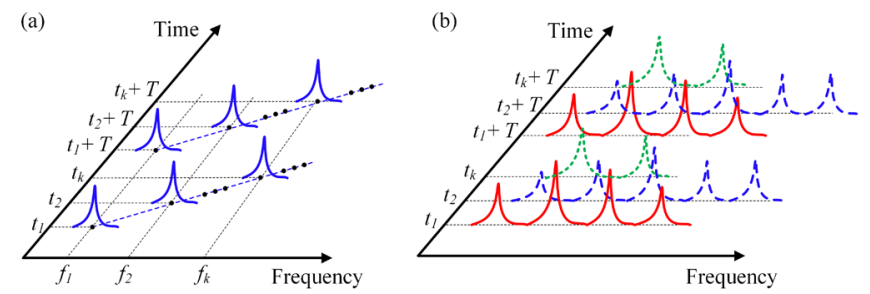

Fig. 3. The operation principle of frequency-scanning filters in (a) the previously reported FDML OEOs and (b) the proposed FDML OEO. T is the period of the optical waveform and also the period of the time-varying filter.

Fig. 3 shows the operation principle of the frequency-scanning filter in the previously reported FDML OEOs and the proposed FDML OEO, respectively. The frequency-scanning characteristic of the filter is the biggest difference between them. As shown in Fig. 3(a), the scanning characteristic of the filter is that its center frequency changes linearly with time. At time $t_{1}$, the frequency-scanning filter is centered at $f_{1}$. When the time changes from $t_{1}$ to $t_{\mathrm{k}}$, the center of the filter is linearly scanned from $f_{1}$ to $f_{\mathrm{k}}$. After a period of $T$, the filter restarts scanning from $f_{1}$ to $f_{\mathrm{k}}$ again. In fact, the optical waveform sent to the FDML OEO is a linearly-chirped optical waveform with a period of $T$. Under these circumstances, either a single-passband filter, or a dual-passband filter at a certain instant can be constructed, and the center frequency of the passband changes linearly with time according to the linearly-chirped optical waveform. Based on the two kinds of filters in conjunction with the FDML, a linear frequency-scanning OEO can be constructed, which can only be used to generate single-chirp or dual-chirp LCMWs with low phase noise. In comparison, in the proposed FDML OEO for arbitrary microwave waveform generation, a reconfigurable OWG is implemented to generate an arbitrary optical waveform, which can be seen as a series of frequency-scanning optical wavelengths in the frequency domain, and at any instant, the number of wavelengths can be from 0 to infinite, which serves as the optical source for the OEO. One example is shown in Fig. 3(b), at time $t_{1}$, the filter has four passbands corresponding to the four wavelengths at $t_{1}$. When the time changes from $t_{1}$ to $t_{\mathrm{k}}$, the filter has its specific filtering characteristics at each time. For example, at time $t_{2}$, the filter has five passbands, and at time $t_{\mathrm{k}}$, the filter has two passbands. After a period of $T$, the multi-passband filter restarts scanning as it does from $t_{1}$ to $t_{\mathrm{k}}$. Here, $T$ is also the period of the arbitrary optical waveform. In this way, a time-varying multi-passband frequency-scanning MPF with much more complex scanning characteristics, which mainly depends on the arbitrary optical waveform, is constructed. Under the condition of the FDML, a frequency-scanning OEO based on the MPF for the generation of arbitrary microwave waveforms can be implemented.

In detail, we manipulate the frequency-scanning filter in a certain way to control its scanning characteristics to periodically change in a certain trend, for example, linear change, non-linear change, or discrete change. Thus, the generation of such a microwave waveform with only one frequency at a certain instant from the FDML OEO, such as LCMWs, NLCMWs, and FH microwave waveforms, is achieved. For other kinds of waveforms, for example, the phase-coded microwave waveforms, there are multiple frequency components at a certain time, a time-varying frequency-scanning multi-passband MPF, as shown in Fig. 3(b), is implemented with its 
scanning period or its multiple equal to the cavity round-trip time. In this way, arbitrary microwave waveforms with multiple frequencies at a certain instant can also be generated. The format of the generated waveform is mainly determined by the MPF constructed by the arbitrary optical waveform. To form a proper MPF for arbitrary microwave waveform generation, the optical waveform injected into the OEO should be carefully selected.

\section{EXPERIMENTAL RESULTS AND DISCUSSION}

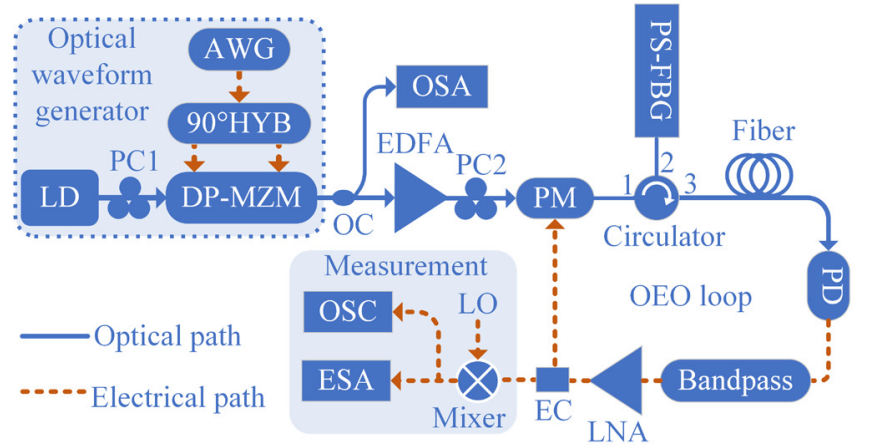

Fig. 4. Arbitrary microwave waveform generation scheme based on a frequency-scanning OEO. LD, laser diode; PC, polarization controller; DP-MZM, dual-parallel Mach-Zehnder modulator; AWG, arbitrary waveform generator; $90^{\circ} \mathrm{HYB}$, $90^{\circ}$ electrical hybrid coupler; EDFA, erbium-doped fiber amplifier; PM, phase modulator; PS-FBG, phase-shifted fiber Bragg grating; PD, photodetector; LNA, low-noise amplifier; EC, electrical coupler; OC, optical coupler; OSA, optical spectrum analyzer; ESA, electrical spectrum analyzer, OSC, oscilloscope; LO, local oscillator.

A proof-of-concept experiment based on the setup shown in Fig. 4 is performed to verify the proposed FDML OEO for arbitrary microwave waveform generation. Firstly, an OWG is constructed as shown in the dotted box in Fig. 4, which mainly consists of a laser diode (LD, ID photonics), a polarization controller (PC), a DP-MZM (Fujitsu 7961EX), an arbitrary waveform generator (AWG, M8195A), and a $90^{\circ}$ electrical hybrid coupler $\left(90^{\circ} \mathrm{HYB}\right.$, Narda 4036C). A 15-dBm continuous-wave (CW) light wave centered at $1549.45 \mathrm{~nm}$ is generated from the LD and injected into the DP-MZM via $\mathrm{PCl}$. The RF signal from the AWG is equally divided into two parts with $90^{\circ}$ phase difference by the $90^{\circ} \mathrm{HYB}$, which are then applied to the two sub-MZMs of the properly-biased DP-MZM to achieve CS-SSB modulation. The CS-SSB modulated optical signal from the DP-MZM is used as the injected arbitrary optical waveform for the FDML OEO. In the experiment, the optical signal is split by a 1:99 optical coupler (OC). The $1 \%$ output from the $\mathrm{OC}$ is connected to an optical spectrum analyzer (OSA, Ando AQ6317B) to monitor the optical spectrum, whereas the output from the $99 \%$ port of the $\mathrm{OC}$ is sent to an EDFA (Amonics, EDFA-PA-35-B) for signal amplification. The optical signal from the EDFA serves as the optical source for the FDML OEO, which is injected into a phase modulator (PM, KG-PM-15-10G-PP-FA) via PC2. Then the output from the PM is injected into a PS-FBG via an optical circulator. The center wavelength of the optical signal from the LD is about $10 \mathrm{GHz}$ away from the narrow notch of the PS-FBG. After CS-SSB modulation at the DP-MZM, the optical sideband, i.e., the input arbitrary optical waveform of the $\mathrm{OEO}$, is about $7 \mathrm{GHz}$ away from the narrow notch by setting the center frequency of the signal from the AWG at 17 GHz. The reflected optical signal from the PS-FBG is sent to a photodetector (PD, Discovery Semiconductors, DCS-40S) with a responsivity of $0.8 \mathrm{~A} / \mathrm{W}$ and a 3-dB bandwidth of $16 \mathrm{GHz}$. The photocurrent from the PD is filtered by an electrical bandpass filter
(KGL, YA354-2, 6.5-8.5 GHz) and amplified by a low-noise amplifier (LNA, CLM/145-7039-293B, 5.85-14.5 GHz) with a 40-dB gain. Finally, the electrical signal from the LNA is divided into two parts by an electrical coupler (EC, KRYTAR, MODEL 1818, $2-18 \mathrm{GHz},-16 \mathrm{~dB}$ ), with most of the electrical signal fed back to the PM to close the OEO loop and the rest part of the electrical signal used for spectrum monitoring. Since the real-time oscilloscope (OSC, Rohde\&Schwarz RTO 2000) in our laboratory has a limited bandwidth, the electrical signal generated from the OEO loop is firstly down-converted to the intermediate frequency band with a 7-dBm local oscillator (LO) signal at around $7 \mathrm{GHz}$ via an electrical mixer (M/A-COM M14A), and then recorded by the OSC and the electrical spectrum analyzer (ESA, Keysight N9020B), respectively. The length of the OEO loop mainly depends on the length of the optical fiber in the OEO. In the experiment, the length of the fiber is about $200 \mathrm{~m}$ and $4.1 \mathrm{~km}$ for two groups of results, respectively.

A. A Conventional Single-Frequency OEO
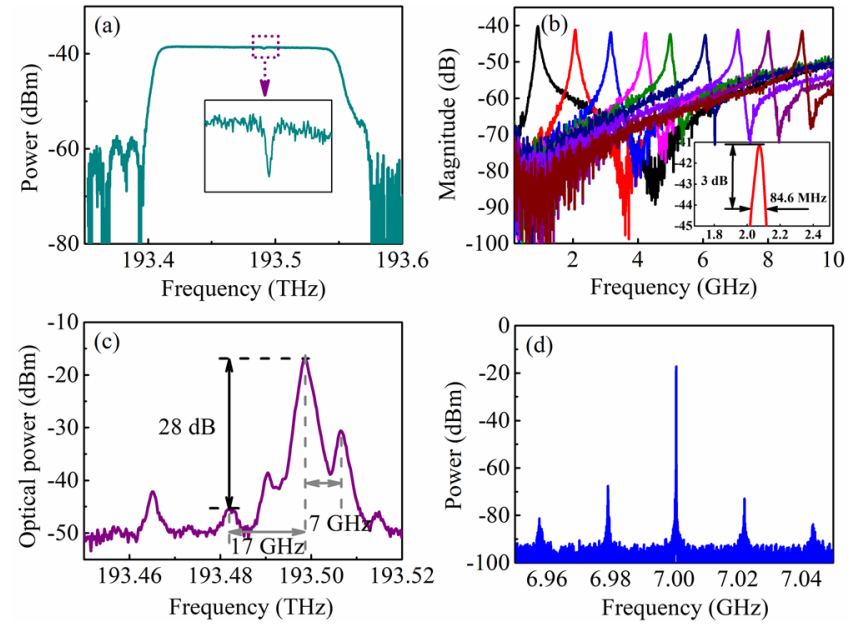

Fig. 5. (a) The reflection spectrum of the PS-FBG, and the inset shows the zoomed-in view of the narrow notch, (b) the frequency response of the tunable MPF, and the inset shows the zoomed-in view of the MPF centered at around $2.05 \mathrm{GHz}$, (c) the optical spectrum at the input of the PD when the stable oscillation in the OEO is established, (d) the corresponding electrical spectrum of the oscillation signal in the OEO without inserting an optical fiber.

A conventional single-frequency OEO based on the setup shown in Fig. 4 is demonstrated. To do so, a 17-GHz RF signal generated from the AWG is sent to the DP-MZM via the $90^{\circ} \mathrm{HYB}$ to achieve the wavelength conversion. The optical wavelength after wavelength conversion serves as the optical source for the OEO. One of the key devices in the proposed OEO is the PS-FBG. The reflection spectrum of the PS-FBG is measured by the OSA and shown in Fig. 5(a), where the inset shows the zoomed-in view of the narrow notch. A vector network analyzer (VNA, Agilent $8720 \mathrm{ES}$ ) is utilized to measure the open-loop response of the $\mathrm{OEO}$, which is also the frequency response of the PS-FBG-based MPF, with the result shown in Fig. 5(b). A tunable MPF with its center frequency varying from 1 to $9 \mathrm{GHz}$ is realized when the wavelength of the LD is tuned. The $3-\mathrm{dB}$ bandwidth of the passband is around $84.6 \mathrm{MHz}$ from the inset. As can be seen from Fig. 5(b), when the center frequency of the MPF increases, the response of the MPF becomes worse, resulting in that only the frequency response below $10 \mathrm{GHz}$ can be used in the experiment. In the experiment, the shifted optical wavelength is around $7 \mathrm{GHz}$ away from the narrow notch of the PS-FBG. Fig. 5(c) shows the measured optical spectrum at the input of the PD when the 
OEO loop is closed and a stable oscillation is established. It can be seen that the wavelength conversion is successfully achieved by CS-SSB modulation and the carrier-suppression ratio is around $28 \mathrm{~dB}$ through finely adjusting the bias voltages of the DP-MZM. More importantly, the two optical sidebands of the shifted optical wavelength are not equal in power, which means the PM-to-IM conversion is achieved due to the notch of the PS-FBG. Fig. 5(d) shows the corresponding electrical spectrum of the oscillation signal in the OEO without inserting an optical fiber. The free spectral range (FSR) is about $20 \mathrm{MHz}$.

Then, FDML OEO is implemented. The key to the FDML OEO implementation is how to accurately match the scanning period of the frequency-scanning filter or its multiple equal to the cavity round-trip time. In fact, the matching accuracy between them plays a vital role in the performance of the FDML OEO. In the experiment, the cavity round-trip time $(\tau)$ is first roughly estimated by the measured FSR of the single-frequency OEO. Then, the scanning period of the frequency-scanning filter is finely adjusted to achieve the best match condition by properly setting the period of driven signal $(T)$ applied to the DP-MZM. For the case with a fiber length of around $200 \mathrm{~m}$, a fundamentally FDML OEO is realized when the period of the driven signal is set to $1.09231 \mu \mathrm{s}(T=\tau)$; for the case with a fiber length of around $4.1 \mathrm{~km}$, a 10th-order harmonically FDML OEO [22] is realized when the period of the driven signal is set to $2.03782 \mu \mathrm{s}$ ( $\mathrm{T}=$ $\tau / 10)$.

B. FDML OEO With a Fiber Length of $200 \mathrm{~m}$

1) LCMW Generation
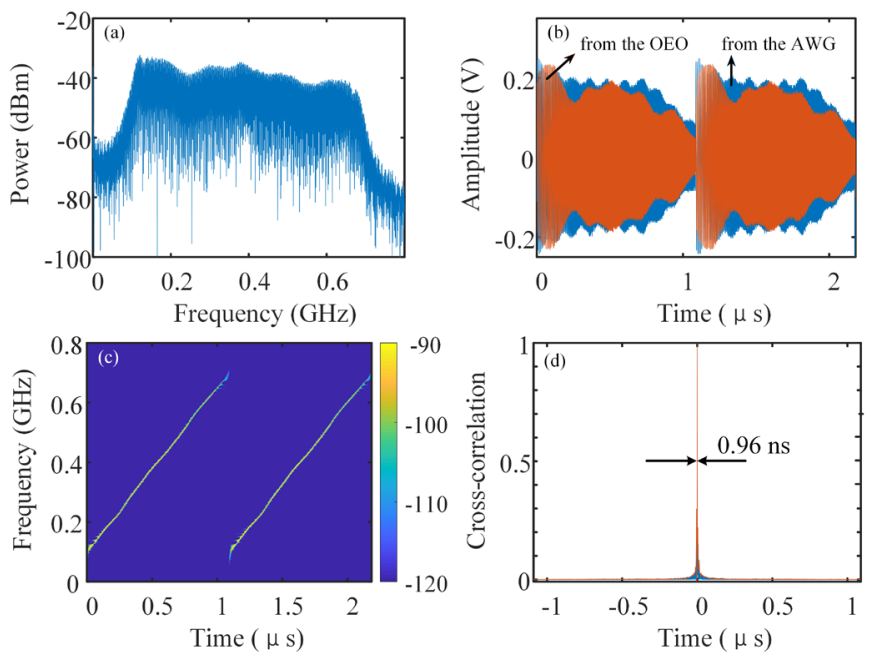

Fig. 6. (a) The electrical spectrum, (b) the temporal waveform, (c) the corresponding time-frequency diagram, and (d) the calculated cross-correlation of the down-converted LCMW from the OEO, with a scanning bandwidth of $600 \mathrm{MHz}$. The blue lines in (b) represent the waveform of the down-converted LCMW from the AWG.

The proposed FDML OEO is firstly utilized to generate the LCMW. Firstly, a linearly-chirped OWG is implemented to generate the arbitrary optical waveform, which serves as the optical source for the FDML OEO. An LCMW with a center frequency of $17 \mathrm{GHz}$ and a bandwidth of $600 \mathrm{MHz}$ from the AWG is applied to the DP-MZM to generate a linearly wavelength-scanning optical waveform through CS-SSB modulation. In this way, a linear frequency-scanning MPF is constructed in the OEO. The scanning period of the MPF is equal to the cavity round-trip time by setting the period of the driving signal to $1.09231 \mu$ s. Fig. 6 shows the experimental results of the down-converted LCMW with a scanning bandwidth of $600 \mathrm{MHz}$. Fig. 6(a) shows the electrical spectrum, while Fig. 6(b) shows the corresponding temporal waveform in two periods. For comparison, the temporal waveform of the down-converted LCMW from the AWG is also shown in Fig. 6(b) in blue lines. From the spectrum and the waveform, an obvious degradation in the high-frequency component is observed from the waveform from the OEO. However, comparing the waveforms from the AWG and the OEO, it is found the degradations of the high-frequency components are roughly consistent with each other, which means that the degradation in the high-frequency component is mainly due to the limited bandwidth of the OSC. The time-frequency diagram can be obtained by the short-time Fourier transform (STFT), with the result shown in Fig. 6(c). As can be seen, the frequency of the oscillation signal in the OEO changes linearly with time, however, with a very small nonlinearity mainly caused by the instability of the PS-FBG-based MPF and the residual mismatch between the tuning rate of the filter (related to the period of the arbitrary optical waveform) and the mode spacing of the OEO (related to the round-trip time of the OEO). Fig. 6(d) shows the cross-correlation of the generated LCMW in two different periods. A compressed narrow pulse is generated, which confirms the good consistency of the generated waveform. The full-width at half maximum (FWHM) of the correlation peak is about $0.96 \mathrm{~ns}$, which indicates that a pulse compression ratio (PCR) of 1137 is achieved. It should be noted that the bandwidth is not the focus of the experiment. Therefore, under the premise to ensure the experimental verification, the maximum bandwidth of the generated signal is $600 \mathrm{MHz}$ in our experiment.

2) $\mathrm{NLCMW}$ Generation
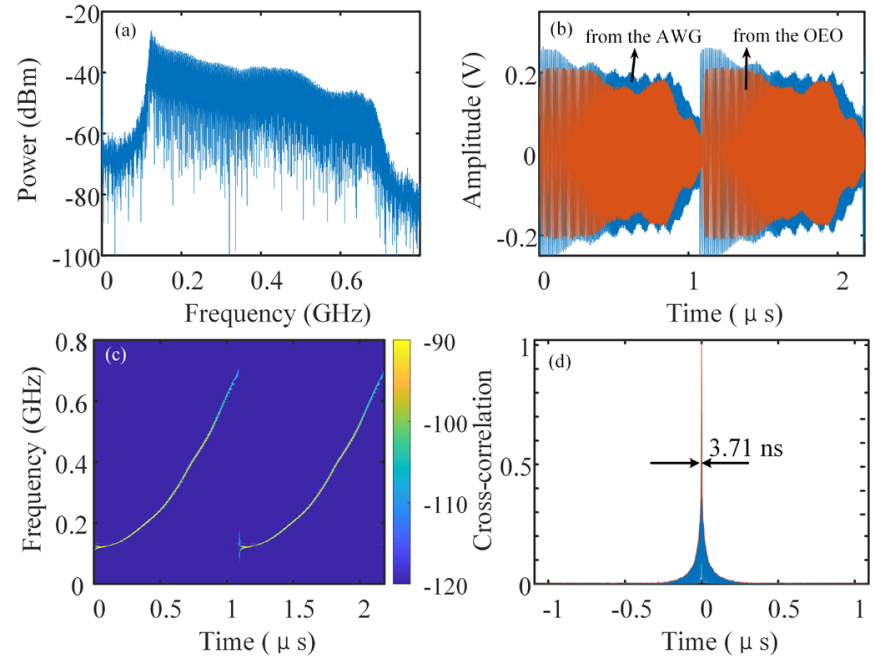

Fig. 7. (a) The electrical spectrum, (b) the temporal waveform, (c) the corresponding time-frequency diagram, and (d) the calculated cross-correlation of the down-converted NLCMW from the OEO, with a scanning bandwidth of $600 \mathrm{MHz}$. The blue lines in (b) represent the waveform of the down-converted NLCMW from the AWG.

To demonstrate the capability of the OEO to generate an arbitrary waveform, the generation of an NLCMW is then studied. An optical waveform must be firstly generated to implement the frequency-scanning MPF for the NLCMW generation. An NLCMW centered at $17 \mathrm{GHz}$ with a scanning bandwidth of $600 \mathrm{MHz}$ and a period of $1.09231 \mu \mathrm{s}$ is generated from the AWG and then applied to the DP-MZM to generate a nonlinear wavelength-scanning optical waveform through CS-SSB modulation, which serves as the optical source for the OEO. In this way, a nonlinear frequency-scanning 
MPF with a scanning period equal to the cavity round-trip time is constructed in the OEO. As expected, an NLCMW with a scanning bandwidth of $600 \mathrm{MHz}$ is generated from the proposed FDML OEO, with the results after frequency down conversion shown in Fig. 7. Fig. 7(a), (b), and (c) show the electrical spectrum, the temporal waveform, and the corresponding time-frequency diagram of the generated NLCMW from the OEO, respectively. For comparison, the temporal waveform of the down-converted NLCMW from the AWG is also shown in blue lines in Fig. 7(b). A degradation at high frequencies is also observed, which is caused by the limited bandwidth of the OSC. Fig. 7(d) shows the cross-correlation of the generated LCMW in two different periods. The FWHM of the correlation peak is about $3.71 \mathrm{ns,}$ which indicates that a PCR of 294.42 is achieved.

3) FH Microwave Waveform Generation
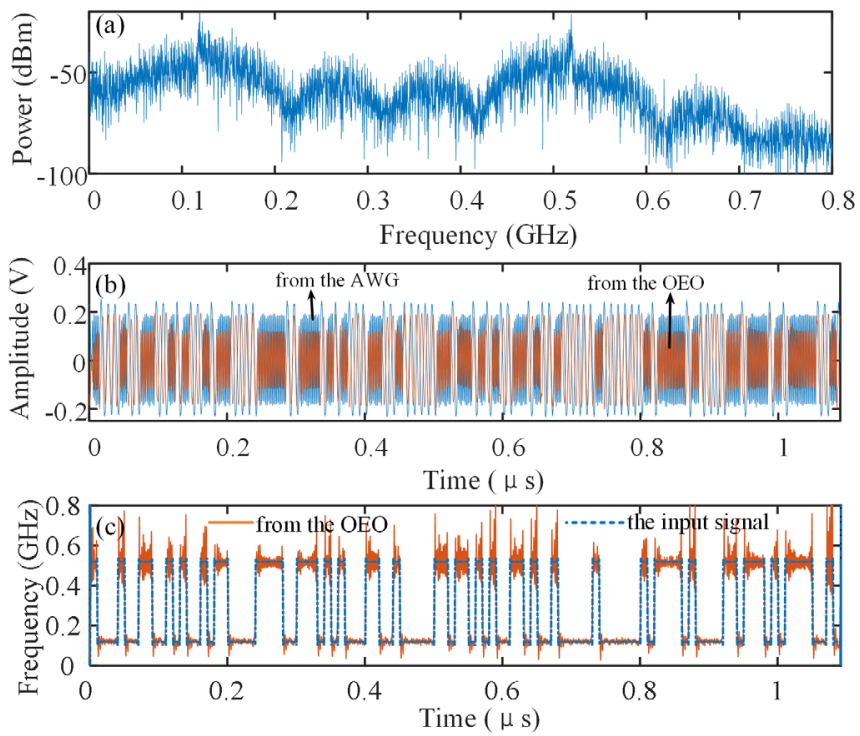

Fig. 8. (a) The electrical spectrum, (b) the temporal waveform, and (c) the corresponding calculated instantaneous frequencies of the down-converted 2-level $\mathrm{FH}$ microwave waveform from the OEO. The temporal waveform in blue in (b) is the down-converted FH microwave waveform from the AWG, and the dotted line in (c) is the calculated instantaneous frequency of the down-converted input signal.

2-level FH microwave waveform with an $\mathrm{FH}$ range of $400 \mathrm{MHz}$ is further generated by the system. In this case, the electrical signal with a period of $1.09231 \mu \mathrm{s}$ from the AWG is applied to the DP-MZM to generate an FH optical waveform, which serves as the optical source for the OEO. Fig. 8 shows the experimental results of the down-converted 2-level FH microwave waveforms generated from the OEO. Fig. 8 (a) shows the electrical spectrum, while Fig. 8(b) shows one period of the temporal waveform. For comparison, the temporal waveform of the down-converted 2-level FH microwave waveform from the AWG is also shown in blue in Fig. 8(b). It is also found that high-frequency components have relatively lower amplitude due to the limited bandwidth of the OSC. The instantaneous frequency of the generated 2-level FH microwave waveform is obtained by Hilbert transform, with the result shown in Fig. 8(c). It is observed that the instantaneous frequencies of the waveform are bouncing between two frequencies and the $\mathrm{FH}$ range is $400 \mathrm{MHz}$. It is also noticed that the instantaneous frequency of the generated FH microwave waveform of the OEO is consistent with that of the down-converted input signal in the blue dotted line. In addition, the FH speed is a key indicator for FH microwave waveforms in radar and communication applications. Here if harmonic mode-locking is used [22], that is, shorten the period of the FH waveform to $1 / \mathrm{n}$ of the loop round-trip time, the FH speed can be further increased. In comparison with the FH microwave waveform generated by a conventional OEO [23], the FH microwave waveform generated using the proposed system does not need extra time to establish a new stable oscillation after the frequency is changed.

4) Phase-Coded Microwave Waveform Generation
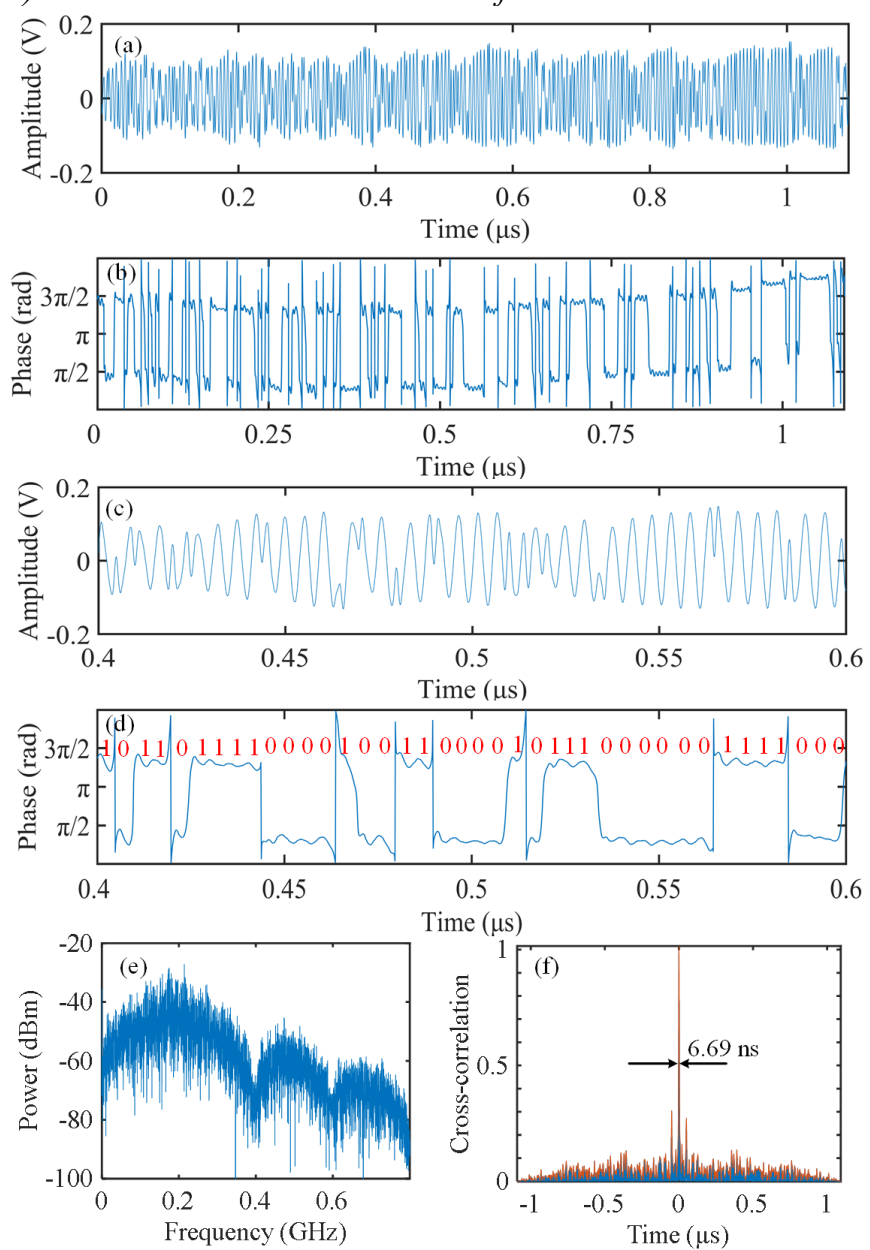

Fig. 9. Experimental results of the down-converted 218-bit binary phase-coded microwave waveform from the OEO. (a) The temporal waveform in one period, (b) the recovered phase information from (a), (c) a section of the generated waveform, (d) the recovered phase information from (c), (e) the electrical spectrum, and (f) the cross-correlation of the down-converted 218-bit binary phase-coded microwave waveform.

To further demonstrate the ability to generate arbitrary microwave waveforms, the phase-coded microwave waveform generation is experimentally verified based on the proposed FDML OEO. Fig. 9 shows the experimental results of the down-converted $1.09231-\mu \mathrm{s}$ 218-bit binary phase-coded microwave waveform from the OEO. Fig. 9(a) shows the temporal waveform in one period, while Fig. 9(b) shows the corresponding recovered phase information by Hilbert transform, which is consistent with the input 218-bit binary sequence (“11000111011001010100010001011010011111001011110101000 011011010001101101000000110101101111000010011000010111 000000111100011011110101100011011111011110000110101111 000000101110100110110000111111000111111101101111111110 101"). Approximate $\pi$ phase shift has been recovered from the 
waveform. Fig. 9(c) and (d) show a section of the generated waveform from 0.4 to $0.6 \mu \mathrm{s}$ and the corresponding recovered phase information. An obvious phase jump can be observed between adjacent "0" and "1" from the waveform. Fig. 9(e) shows the electrical spectrum of the generated phase-coded microwave waveform. Fig. 9(f) shows the cross-correlation of the generated phase-coded microwave waveform in two different periods. The FWHM of the correlation peak is about $6.69 \mathrm{~ns}$, which indicates that a PCR of 163 is achieved.
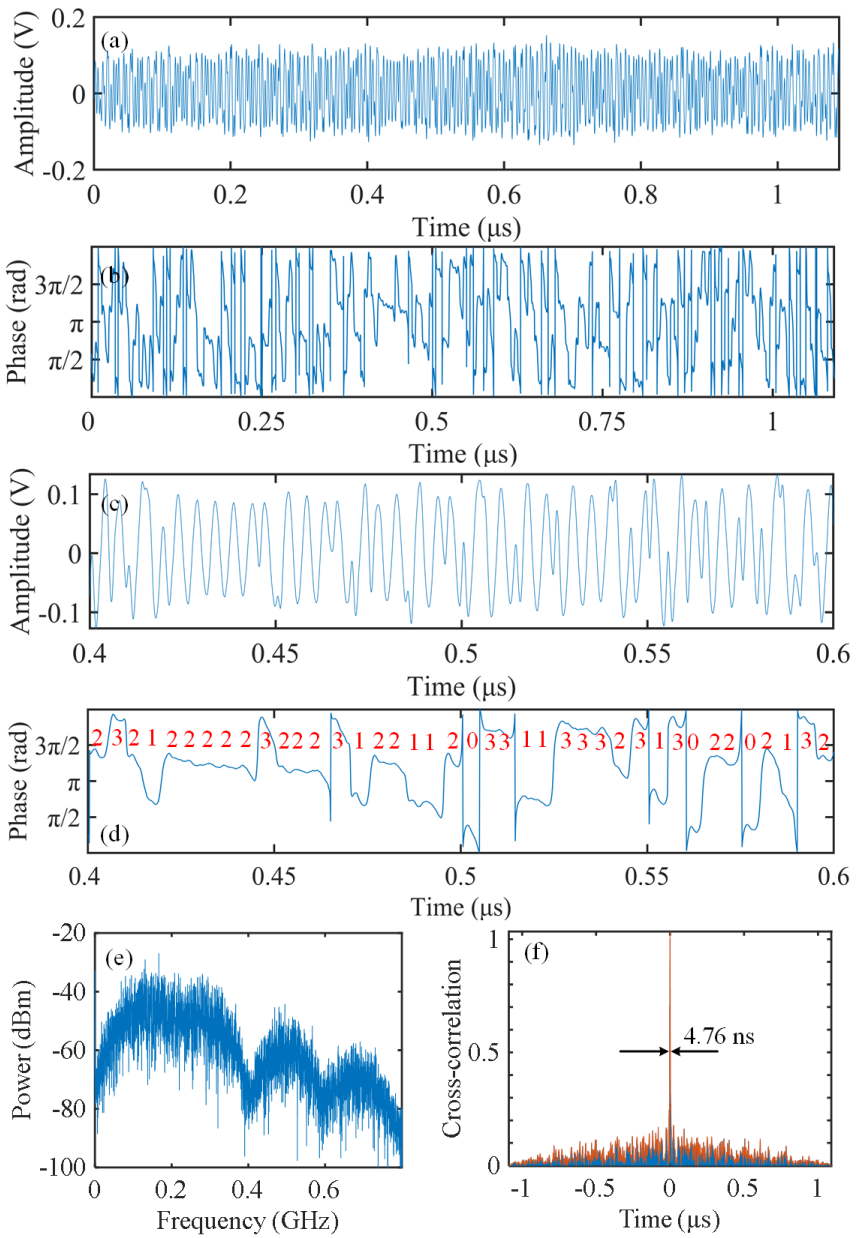

Fig. 10. Experimental results of the down-converted 218-bit quaternary phase-coded microwave waveform from the OEO. (a) The temporal waveform in one period, (b) the recovered phase information from (a), (c) a section of the generated waveform, (d) the recovered phase information from (c), (e) the electrical spectrum, and (f) the cross-correlation of the down-converted 218-bit quaternary phase-coded microwave waveform.

218-bit quaternary phase-coded microwave waveforms with the same period of $1.09231 \mu \mathrm{s}$ are further generated from the OEO. Fig. 10 shows the experimental results of down-converted 218-bit quaternary phase-coded microwave waveforms. Fig. 10(a) shows the temporal waveform in one period, while Fig. 10(b) shows the corresponding recovered phase information, which is consistent with the input 218-bit sequence (“"01321230232012010131203012302320011101321300301100112 031023131103010113322023110232122222322231221120331133 323130220213233201032321032013221001221312111322002300 031131220213202012323023020120323212102312202030021021 131"). Fig. 10(c) and (d) show a section of the generated waveform from 0.4 to $0.6 \mu$ s and the corresponding recovered phase information. Fig. 10(e) shows the electrical spectrum of the waveform. Fig. 10(f) shows the cross-correlation of the generated phase-coded microwave waveform in two different periods. The FWHM of the correlation peak is about $4.76 \mathrm{~ns}$, which indicates that a PCR of 229 is achieved.

\section{FDML OEO With a Fiber Length of $4.1 \mathrm{~km}$}
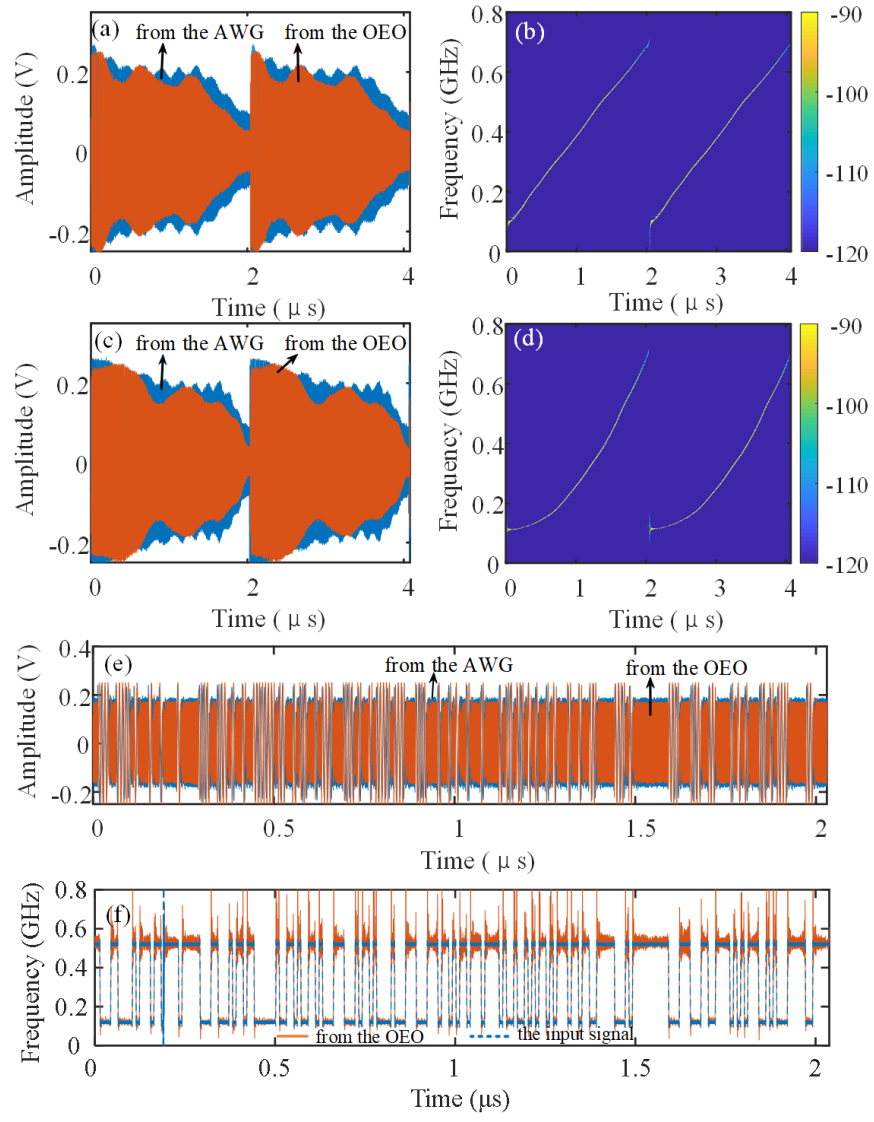

Fig. 11. Experimental results of the down-converted LCMW, NLCMW, and FH microwave waveform when the length of the fiber is changed to $4.1 \mathrm{~km}$. (a), (c), (e) The temporal waveforms of the LCMW, the NLCMW, and the FH microwave waveform from the $\mathrm{OEO}$, (b), (d) the corresponding time-frequency diagrams of the LCMW and the NLCMW, (f) the calculated instantaneous frequencies of the FH microwave waveform. The temporal waveforms in blue in (a), (c), and (e) are the down-converted LCMW, NLCMW, and FH microwave waveform from the AWG, respectively, and the dotted line in (f) is the calculated instantaneous frequency of the down-converted input signal.

The loop length determines the $\mathrm{Q}$ factor of the OEO, which directly influences the phase-noise of the generated signal. Therefore, the length of the fiber in the FDML OEO is changed to $4.1 \mathrm{~km}$. In this case, the generation of arbitrary microwave waveform is further studied. The period of the driven signal is finely adjusted to achieve the best matching and finally determined as $2.03782 \mu$ s to implement a 10th-order harmonic mode-locking. Fig. 11 shows the experimental results of the down-converted LCMW, NLCMW, and FH microwave waveform generated from the OEO. The waveforms of the generated LCMW, NLCMW, and FH microwave waveform are shown in Fig. 11(a), (c), and (e), and the corresponding time-frequency diagrams and instantaneous frequency are shown in Fig. 11(b), (d), and (f). The down-converted waveforms from the AWG are also shown in Fig. 11 in blue lines. The degradation of 
high-frequency components due to the limited bandwidth of the OSC is also observed. In addition, because the length of the fiber is about $4.1 \mathrm{~km}$, in this case, the mode spacing of the OEO is much smaller than that when the length of the fiber is $200 \mathrm{~m}$. However, the 3-dB bandwidth of the MPF is fixed to around $84.6 \mathrm{MHz}$, which is determined by the PS-FBG used in the experiment. Under these circumstances, the single-mode oscillation at any instant in the OEO is much more difficult to be guaranteed due to the much smaller mode spacing, thus the frequency stability of the arbitrary waveform generated from the FDML OEO is reduced compared with the results using a 200-m fiber. Nevertheless, the frequency variation characteristics of the LCMW, NLCMW, and FH microwave waveform can be seen.
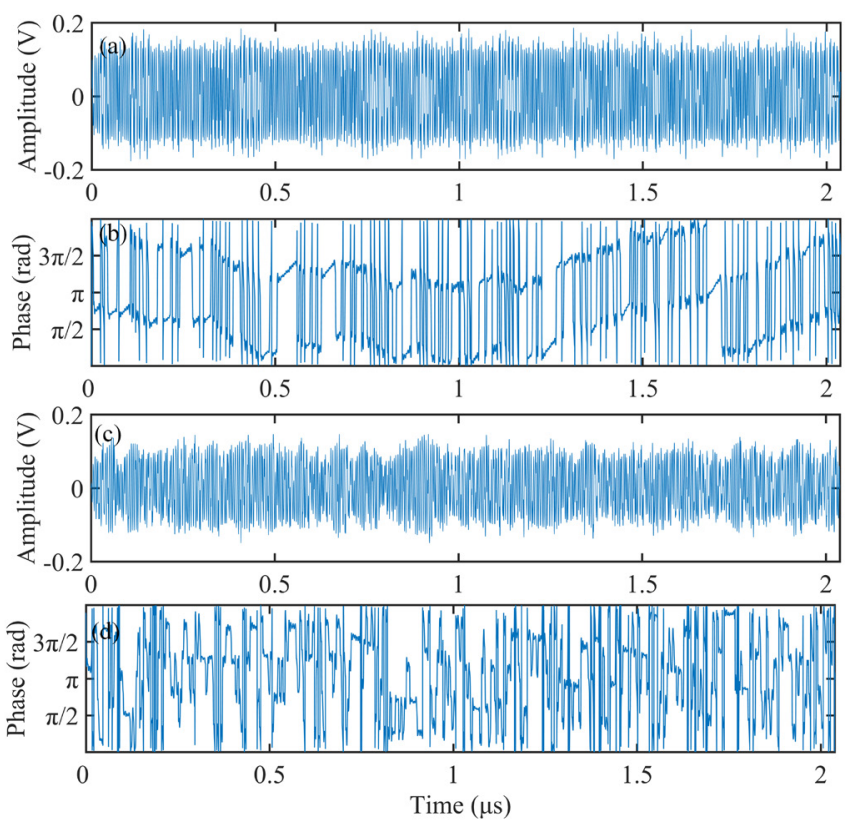

Fig. 12. Experimental results of the down-converted 218-bit binary and quaternary phase-coded microwave waveform from the $\mathrm{OEO}$ when the length of the fiber is changed to $4.1 \mathrm{~km}$. (a), (c) The temporal waveforms, (b), (d) the corresponding recovered phase information.

Phase-coded microwave waveform generation is also verified in this case. Fig. 12 shows the experimental results of down-converted 218-bit binary phase-coded microwave waveform and quaternary phase-coded microwave waveform. Fig. 12(a) and (c) show the temporal waveforms, while the corresponding recovered phase information is shown in Fig. 12 (b) and (d), respectively. The frequency instability will have a greater impact on the phase information recovery of the phase-coded microwave waveform. As shown in Fig. 12(b) and (d), approximate $\pi / 2, \pi$, or $3 \pi / 2$ phase shift between adjacent codes has been recovered from the waveforms. However, from the waveform in a period, the recovered phase information is not as good as the results using a $200-\mathrm{m}$ fiber. The main reason for this phenomenon is the frequency instability caused by the mode competition introduced by the limited bandwidth of MPF. If a PS-FBG with a much narrower bandwidth and better stability is used in the experiment to select the oscillation mode more accurately, the performance of the generated arbitrary waveforms can be greatly improved in this case.

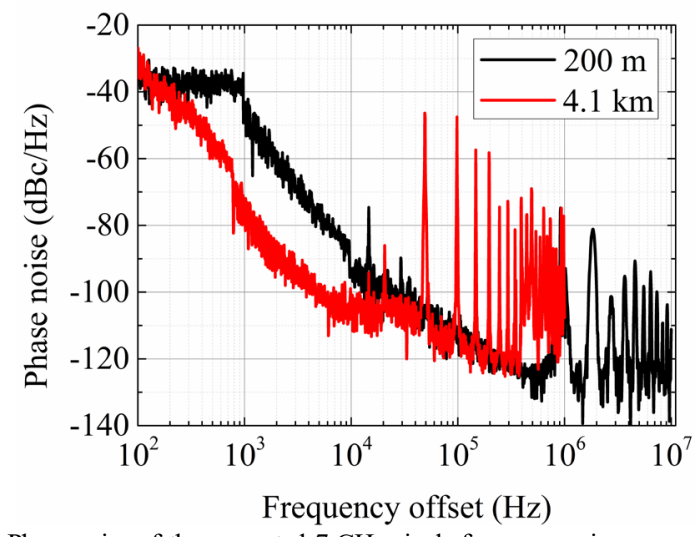

Fig. 13. Phase noise of the generated 7-GHz single-frequency microwave waveform from the $\mathrm{OEO}$ with a fiber length of $200 \mathrm{~m}$ and $4.1 \mathrm{~km}$.

Fig. 13 shows the phase noise of the 7-GHz oscillation signal generated by the FDML OEO, which is measured by the ESA. As shown by the red curve, the phase noise is around $-95 \mathrm{dBc} / \mathrm{Hz}$ at $10-\mathrm{kHz}$ frequency offset for the case with a fiber length of $200 \mathrm{~m}$. The mode spacing is about $917 \mathrm{kHz}$, which is defined by the cavity round-trip time. When the length of the OEO loop is increased by using a long fiber of $4.1 \mathrm{~km}$, the phase noise is reduced to about -108 $\mathrm{dBc} / \mathrm{Hz}$ at $10-\mathrm{kHz}$ frequency offset. The mode spacing is about 49 $\mathrm{kHz}$ in this case. As can be seen, when the length of the fiber is changed from $200 \mathrm{~m}$ to $4.1 \mathrm{~km}$, the phase noise of the OEO is greatly reduced when the frequency offset is within $10 \mathrm{kHz}$. When the frequency offset is larger than $10 \mathrm{kHz}$, the phase noise is comparable to the case with a $200-\mathrm{m}$ fiber length. The limitation to achieve a better phase noise for the OEO with a 4.1-km fiber length is mainly due to the fabrication of the PS-FBG, which has a bandwidth that can cover too many eigenmodes. In fact, using the PS-FBG with a notch of $84.6 \mathrm{MHz}$, the $\mathrm{OEO}$ is very difficult to oscillate stably, as shown in Fig. 11, where the frequencies of the generated arbitrary microwave waveforms are not very stable. However, this problem can be solved by using a better fabricated PS-FBG. In addition, from the datasheet of the AWG used in our experiment, the phase noise of the $10 \mathrm{GHz}$ signal from the AWG is around $-96 \mathrm{dBc} / \mathrm{Hz},-103 \mathrm{dBc} / \mathrm{Hz}$, and -116 $\mathrm{dBc} / \mathrm{Hz}$ at $10 \mathrm{kHz}, 100 \mathrm{kHz}$, and $1 \mathrm{MHz}$ frequency offsets, respectively. We can roughly calculate the phase noise of the 7-GHz signal to $-99 \mathrm{dBc} / \mathrm{Hz},-106 \mathrm{dBc} / \mathrm{Hz}$, and $-119 \mathrm{dBc} / \mathrm{Hz}$ at $10 \mathrm{kHz}, 100$ $\mathrm{kHz}$, and $1 \mathrm{MHz}$ frequency offsets, respectively. Compared with our results in Fig. 13, the OEO does improve the phase noise performance of the generated signal. At a lower offset frequency that represents the relatively long-term stability, the phase noise of the generated signal is not as good as that from the AWG, which is due to the relatively poor long-term stability of the PS-FBG and LD and can be improved by photonic integration and better laser control. Therefore, we can expect to use the proposed system to generate arbitrary microwave waveforms with better phase noise performance.

\section{Discussion}

The experimental results above indicate that arbitrary waveform generation based on the proposed FDML OEO is achieved. However, some more issues need to be further discussed and explained. 


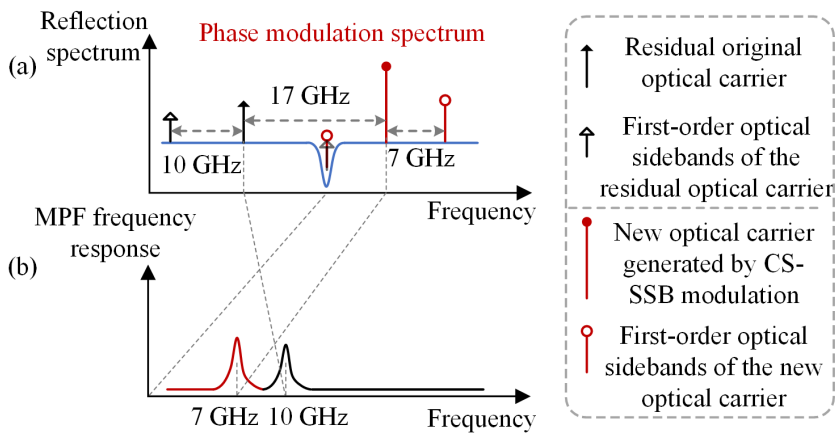

Fig. 14. (a) The reflection spectrum of the PS-FBG, (b) the frequency response of the dual-passband MPF due to the residual optical carrier.

Similar to the generation of LCMWs by using a linear wavelength-scanning optical waveform, arbitrary microwave waveforms are generated based on the proposed FDML OEO by using a corresponding arbitrary optical waveform. In our experiment, the generated signal from the OEO is similar to that from the AWG. In fact, the arbitrary optical waveform can be generated by many other methods without using an electrical arbitrary waveform from an AWG, for example, directly modulating a laser diode using a triangle-wave or sawtooth currents. We use the AWG because modulating an optical carrier using a waveform generated from an AWG is the simplest way for us to realize the arbitrary optical waveform for the FDML-OEO. Other methods to realize the time-varying multi-passband frequency-scanning filter for arbitrary waveform generation are very important to simplify the system and need to be further studied in the future. Nevertheless, the FDML OEO is to map the arbitrary optical waveforms in the optical domain to an electrical arbitrary waveform in the electric domain by using the key feature of low phase noise offered by the OEO. Therefore, the most important thing is providing an arbitrary optical waveform. In this paper, the external CS-SSB modulation is employed to implement the OWG, serving as the optical source for the frequency-scanning OEO, as shown in Fig. 14. The optical carrier from the LD is about $10 \mathrm{GHz}$ away from the narrow notch of the PS-FBG, which is CS-SSB modulated by the electrical signal centered at $17 \mathrm{GHz}$ from the AWG. As can be seen, the first-order optical sideband of the carrier is around $7 \mathrm{GHz}$ away from the notch, which is used as the arbitrary optical waveform for the FDML OEO. However, due to the non-ideal extinction ratio and the bias drift of the modulator, the CS-SSB modulated optical signal still has a residual optical carrier, which cannot be filtered out in our experiment due to the lack of a suitable optical filter. When such an optical signal is injected into the OEO loop, the first-order optical sideband and the residual optical carrier will form two individual MPFs, which influences the generation of the desired arbitrary microwave waveform. Furthermore, the first-order optical sideband and the residual optical carrier will beat each other, which will take a large part of the output power of the LNA, thus influencing the generation of the desired arbitrary microwave waveform. To avoid the influence from the residual optical carrier, an electrical bandpass filter (KGL, YA354-2, 6.5-8.5 $\mathrm{GHz}$ ) is used in the system to filter out the unwanted frequency components.

In the experiment, arbitrary microwave waveforms with different formats are generated at around $7 \mathrm{GHz}$. Several reasons limit the frequency of the generated arbitrary waveforms in the experiment: 1) limited by the fabrication, the PS-FBG can only form a usable MPF with a center frequency less than $10 \mathrm{GHz} ; 2$ ) the LNA that is used in the experiment has a bandwidth from 5.85 to $14.5 \mathrm{GHz}$; 3) the method to implement the OWG needs an electrical bandpass filter to eliminate other interference components as shown in Fig. 13, and the appropriate one in our laboratory is from 6.5-8.5 GHz. For the bandwidth of the generated arbitrary microwave waveforms, a maximum bandwidth of $600 \mathrm{MHz}$ is demonstrated. The bandwidth in our experiment is mainly limited by the bandwidth of the OSC used in the experiment, which is also the reason why the generated signal is down-converted to less than $1 \mathrm{GHz}$ before waveform capture.

The stability of the generated arbitrary waveform largely depends on the wavelength stability of the laser source, the response and

TABLE I

COMPARISON OF DIFFERENT FDML OEOS

\begin{tabular}{|c|c|c|c|c|c|c|}
\hline & Type & Filter Used & $\begin{array}{l}\text { Implementation of the } \\
\text { Frequency-Scanning Filter }\end{array}$ & $\begin{array}{l}\text { Generated } \\
\text { Waveform }\end{array}$ & $\begin{array}{c}\text { Demonstrated } \\
\text { Bandwidth } \\
(\mathrm{GHz})\end{array}$ & $\begin{array}{c}\text { Frequency } \\
\text { Multiplication }\end{array}$ \\
\hline Ref. [9] & \multirow{2}{*}{$\begin{array}{c}\text { Based on a } \\
\text { single-frequency } \\
\text { laser source and a } \\
\text { frequency-scanni } \\
\text { ng electrical filter }\end{array}$} & \multirow{2}{*}{$\begin{array}{l}\text { Single-passband } \\
\text { electrical filter }\end{array}$} & $\begin{array}{l}\text { Tunable RF source with frequency } \\
\text { converter pair }\end{array}$ & \multirow{7}{*}{$\begin{array}{l}\text { Single-chirp } \\
\text { LCMWs }\end{array}$} & 1 & \multirow{4}{*}{ No } \\
\hline Ref. [20] & & & Diode-tuned electrical phase shifter & & 0.4 & \\
\hline Ref. [14] & \multirow{6}{*}{$\begin{array}{l}\text { Based on an } \\
\text { optical waveform } \\
\text { generator and a } \\
\text { reconfigurable } \\
\text { frequency-scanni } \\
\text { ng MPF }\end{array}$} & \multirow{5}{*}{$\begin{array}{c}\text { Single-passband } \\
\text { MPF }\end{array}$} & Tunable laser with SBS deamplification & & 4.5 & \\
\hline Ref. [15] & & & CS-SSB modulation with SBS & & 4 & \\
\hline Ref. [19] & & & $\begin{array}{l}\text { High-order CS-SSB modulation with } \\
\text { SBS }\end{array}$ & & 8 & \multirow{3}{*}{ Yes } \\
\hline Ref. [12] & & & Optical injection & & 15.6 & \\
\hline Ref. $[17]^{*}$ & & & Tunable laser with PS-FBG & & 6 & \\
\hline This work & & $\begin{array}{c}\text { Time-varying } \\
\text { multi-passband } \\
\text { MPF }\end{array}$ & CS-SSB modulation with PS-FBG & $\begin{array}{l}\text { Arbitrary } \\
\text { waveform } \\
\text { generation } \\
\end{array}$ & 0.6 & No \\
\hline
\end{tabular}

*In addition to generate dual-chirp LCMWs, the methods in Ref. [17] can also be utilized to generate single-chirp LCMWs by only using one LD. Note that the frequency and bandwidth doubling are only for the generation of single-chirped LCMWs. 
stability of the PS-FBG, and the stability of the fiber. In the experiment, we need to turn off the dither control of the LD to successfully implement the PS-FBG-based MPF, but this will bring the drift of the laser wavelength. The stability of FBG is affected not only by the manufacturing but also the external factors such as temperature and stress. Therefore, in practice, isolating the PS-FBG to eliminate these influences is necessary to further improve the stability of the system. Furthermore, as we discussed above, the bandwidth of the narrow notch is about $84.6 \mathrm{MHz}$, which leads to serious mode competition, especially when the length of the fiber is long. The fiber is also easily influenced by environmental changes, which also influences the stability of the generated arbitrary waveforms. Therefore, if a long optical fiber is used in the OEO loop, a length compensation or environmental control can greatly increase the long-term stability of the generated arbitrary waveform.

Furthermore, in the experiment, the period of the arbitrary optical waveform is adjusted to make the OEO deviate away from the best condition for FDML. It can be seen that, with the increase of the period deviation of the arbitrary optical waveform from the best value in the experiment, the quality of the oscillation arbitrary microwave waveform in the OEO will become worse and worse. When the deviation reaches a certain value, the oscillation in the $\mathrm{OEO}$ cannot be maintained. Besides, the larger the bandwidth of the generated arbitrary microwave waveform from the $\mathrm{OEO}$, the smaller the deviation needed to make the $\mathrm{OEO}$ unable to oscillate.

Table 1 shows a comparison of the FDML OEOs reported before with the proposed FDML OEO in this paper. As mentioned before, there are two implementation schemes for the FDML OEO. The methods proposed in [9] and [20] belong to the first one using frequency scanning electrical filters, while others belong to the second one using MPFs. For the methods using an MPF constructed via a wavelength scanning optical arbitrary waveform, the LCMW with low phase noise is generated by converting the wavelength scanning optical waveform to the electrical domain with the help of a frequency-scanning OEO based on FDML. However, the previously reported methods are only reported to generate different kinds of LCMWs. The proposed scheme in this paper report the arbitrary microwave waveform generation based on the FDML OEO for the first time, which provides a promising solution to utilize the low phase noise merit of an OEO to generate low phase noise arbitrary microwave waveforms.

\section{CONCLUSION}

In summary, an FDML OEO for arbitrary microwave waveform generation is proposed and experimentally demonstrated. The key contribution of the work is that the concept of FDML OEO is further analyzed and extended to generate arbitrary microwave waveforms. To the best of our knowledge, this is the first time that an OEO is demonstrated for arbitrary microwave waveform generation. An experiment is performed. LCMWs, NLCMWs, FH microwave waveforms, and phase-coded microwave waveforms are generated from the frequency-scanning OEO with a fiber length of $200 \mathrm{~m}$ and $4.1 \mathrm{~km}$. Based on the proposed system, in addition to the single-frequency microwave signal and LCMWs, low phase noise arbitrary microwave waveforms can be generated utilizing the low phase noise merit of an OEO.

\section{REFERENCES}

[1] J. Capmany and D. Novak, "Microwave photonics combines two worlds," Nat. Photon., vol. 1, no. 6, pp. 319-330, Jun. 2007.

[2] D. Marpaung, C. Roeloffzen, R. Heideman, A. Leinse, S. Sales, and J. Capmany, "Integrated microwave photonics," Laser Photon. Rev., vol. 7, no. 4, pp. 506-538, Jul. 2013.

[3] J. Yao, "Microwave Photonics," J. Lightw. Technol., vol. 27, no. 3, pp. 314-335, Feb. 2009.

[4] X. Zou, B. Lu, W. Pan, L. Yan, A. Stöhr, and J. Yao, "Photonics for microwave measurement," Laser Photonics Rev., vol. 10, no. 5, pp. 711-734, Sept. 2016.

[5] K. Xu, R. Wang, Y. Dai, F. Yin, J. Li, Y. Ji, and J. Lin, "Microwave photonics: radio-over-fiber links, systems, and applications," Photon. Res., vol. 2, no. 4, pp. B54-B63, Aug. 2014.

[6] T. Berceli and P. R. Herczfeld, "Microwave Photonics-A Historical Perspective," IEEE Trans. Microw. Theory Tech., vol. 58, no. 11, pp. 2992-3000, Nov. 2010.

[7] J. Yao, "Photonic generation of microwave arbitrary waveforms," Opt. Commun., vol. 284, no. 15, pp. 3723-3736, Jul. 2011.

[8] X. S. Yao and L. Maleki, "Optoelectronic microwave oscillator," J. Opt. Soc. Am. B, vol. 13, no. 8, pp. 1725-1735, Aug. 1996.

[9] Q. Cen, Y. Dai, F. Yin, Y. Zhou, J. Li, J. Dai, L. Yu, and K. Xu, "Rapidly and continuously frequency-scanning opto-electronic oscillator," Opt. Express, vol. 25, no. 2, pp. 635-643, Jan. 2017.

[10] T. Hao, Q. Cen, Y. Dai, J. Tang, W. Li, J. Yao, N. Zhu, and M. Li, "Breaking the limitation of mode building time in an optoelectronic oscillator," Nat. Commun., vol. 9, May 2018, Art. no. 1839.

[11] R. Huber, M. Wojtkowski, and J. G. Fujimoto, "Fourier domain mode locking (FDML): a new laser operating regime and applications for optical coherence tomography," Opt. Express, vol. 14, no. 8, pp. 3225-3237, Apr. 2006.

[12] P. Zhou, F. Z. Zhang, and S. L. Pan, "Generation of Linear Frequency-Modulated Waveforms by A Frequency-Sweeping Optoelectronic Oscillator," J. Lightwave Technol., vol. 36, no. 18, pp. 3927-3934, Sept. 2018.

[13] T. Hao, J. Tang, W. Li, N. Zhu, and M. Li, "Tunable Fourier Domain Mode-Locked Optoelectronic Oscillator Using Stimulated Brillouin Scattering," IEEE Photon. Technol. Lett., vol. 30, no. 21, pp. 1842-1845, Nov. 2018.

[14] T. Hao, J. Tang, W. Li, N. Zhu, and M. Li, "Fourier domain mode locked optoelectronic oscillator based on the deamplification of stimulated Brillouin scattering," OSA Continuum, vol. 1, no. 2, pp. 408-415, Oct. 2018.

[15] Z. Zeng, L. Zhang, Y. Zhang, Z. Zhang, S. Zhang, Y. Zhang, B. Sun, and Y. Liu, "Frequency-definable linearly chirped microwave waveform generation by a Fourier domain mode locking optoelectronic oscillator based on stimulated Brillouin scattering," Opt. Express, vol. 28, no. 9, pp. 13861-13870, Apr. 2020.

[16] T. Hao, J. Tang, N. Shi, W. Li, N. Zhu, and M. Li, "Dual-chirp Fourier domain mode-locked optoelectronic oscillator," Opt. Lett., vol. 44, no. 8, pp. 1912-1915, Apr. 2019.

[17] S. Zhu, X. Fan, B. Xu, W. Sun, M. Li, N. Zhu, and W. Li, "Polarization Manipulated Fourier Domain Mode-Locked Optoelectronic Oscillator," J. Lightwave Technol., vol. 38, no. 19, pp. 5270-5277, Oct. 2020.

[18] L. Zhang, Z. Zeng, Y. Zhang, Z. Zhang, S. Zhang, G. Ni, and Y. Liu, "Frequency-Sweep-Range-Reconfigurable Complementary Linearly Chirped Microwave Waveform Pair Generation by Using a Fourier Domain Mode Locking Optoelectronic Oscillator Based On Stimulated Brillouin Scattering," IEEE Photon. J., vol. 12, no. 3, Jun. 2020, Art. no. 5501010.

[19] H. Zhang, F. Zhang, S. Pan, X. Ye, S. Liu, and H. Chen, "Photonic Generation of Linearly Chirped Microwave Waveforms With Tunable Parameters," IEEE Photon. Technol. Lett., vol. 32, no. 17, pp. 1037-1040, Sept. 2020.

[20] P. Hao, H. Lu, R. Han, X. Wang, X. Liu, and X. S. Yao, "Fourier domain mode-locked opto-electronic oscillator with a diode-tuned bandpass filter," Opt. Express, vol. 28, no. 16, pp. 23454-23466, Aug. 2020.

[21] B. Yang, H. Zhao, Z. Cao, S. Yang, Y. Zhai, J. Ou, and H. Chi, "Active mode-locking optoelectronic oscillator," Opt. Express, vol. 28, no. 22, pp. 33220-33227, Oct. 2020.

[22] T. Hao, J. Tang, W. Li, N. Zhu, and M. Li, "Harmonically Fourier Domain Mode-Locked Optoelectronic Oscillator," IEEE Photon. Technol. Lett., vol. 31, no. 6, pp. 427-430, Mar. 2018.

[23] W. Li, W. Zhang, and J. Yao, "Frequency-hopping microwave waveform generation based on a frequency-tunable optoelectronic oscillator," in Optical Fiber Communication Conference, OSA Technical Digest (online) (Optical Society of America, 2014), paper W1J.2. 